University of Nebraska - Lincoln

DigitalCommons@University of Nebraska - Lincoln

August 1984

\title{
Influence of crystal fields on the quasimetallic reflection spectra of crystals: Optical spectra of polymorphs of a squarylium dye
}

\author{
M. Tristani-Kendra \\ University of Nebraska - Lincoln \\ Craig J. Eckhardt \\ University of Nebraska - Lincoln, ceckhardt1@unl.edu
}

Follow this and additional works at: https://digitalcommons.unl.edu/chemistryeckhardt

Part of the Chemistry Commons

Tristani-Kendra, M. and Eckhardt, Craig J., "Influence of crystal fields on the quasimetallic reflection spectra of crystals: Optical spectra of polymorphs of a squarylium dye" (1984). Craig J. Eckhardt Publications. 30.

https://digitalcommons.unl.edu/chemistryeckhardt/30

This Article is brought to you for free and open access by the Published Research - Department of Chemistry at DigitalCommons@University of Nebraska - Lincoln. It has been accepted for inclusion in Craig J. Eckhardt Publications by an authorized administrator of DigitalCommons@University of Nebraska - Lincoln. 


\title{
Influence of crystal fields on the quasimetallic reflection spectra of crystals: Optical spectra of polymorphs of a squarylium dye $e^{\text {a) }}$
}

\author{
M. Tristani-Kendrab) and C. J. Eckhardt ${ }^{\text {c) }}$ \\ Department of Chemistry, University of Nebraska-Lincoln, Lincoln, Nebraska 68588-0304
}

(Received 27 December 1983; accepted 10 April 1984)

\begin{abstract}
The reflection and Kramers-Kronig absorption spectra have been obtained from the monoclinic and triclinic polymorphs of a squarylium dye, 2,4-bis (4-diethylamino-2-hydroxy phenyl) cyclobutadienediylium-1,3-diolate. The extremely different optical responses were found to arise from two molecular singlet transitions of essentially long axis polarization. Successful application of a four oscillator molecular exciton-polariton theory required the use of point charge densities rather than point dipoles in an extension of the theory which employed both interactions between the two singlets as well as a frequency dependent lattice damping. An intermolecular charge transfer transition is also assigned.
\end{abstract}

\section{INTRODUCTION}

The optical spectroscopy of interacting dye molecules, whether of their aggregates found at high molecular concentrations in solution or of single crystals, has been of continued interest for nearly five decades. ${ }^{1}$ In solution the intense, red shifted $J$ band is the most striking manifestation of collective interaction while in the crystal the appearance of quasimetallic reflection bands of high reflectivity and widths on the order of an electron volt are the hallmark of the strong coupling observed in these systems. Much effort has been expended in the study of these systems in attempts to understand the large changes in bandshape and shifts in frequencies from those observed in the free molecule. ${ }^{2}$

The excitonic origin of these spectra affords an effective approach to analysis but, because the coupling is strong and the formation of aggregates and single crystals is often unpredictable, the details, both experimental and theoretical, have not been rapidly developed. In the case of aggregates in solution, major questions exist regarding the structure of the aggregates as well as their optical response. Although structure is not a problem with the dye crystal, study is hampered by the resistance of spectra to sharpening at low temperatures. Thus, complete description of the spectra of the aggregates and crystals has proved to be elusive. Part of this diffculty arises from a paucity of detailed experimental results .

Correlation of the structure of $J$-band aggregates to the arrangement of molecules in crystals which have spectra similar to that of the aggregate have been attempted. ${ }^{3}$ These studies, while quite illuminating, have not been as conclusive as desired because total agreement between crystal and aggregate spectra was not achieved. Often, a given dye will have polymorphs and an exhaustive characterization of crystal structure and spectra may be required to obtain the requisite spectral agreement. Part of the difficulty in obtaining a match between the crystal and aggregate spectra may arise from failure to obtain spectra from that polymorph

\footnotetext{
a) Supported by DOE Grant No. DE-FG-02-79ER 10535 and in part by NSF Grant No. DMR-7908759.

b) Work completed in partial fulfillment of requirements for the Ph.D. at the University of Nebraska.

c) Author to whom correspondence should be addressed.
}

whose packing would most mimic the structure of the aggregate.

There is also a lack of structure-spectra studies among various dye polymorphic crystals and, without this information, attempts to understand the more complicated problem of dye aggregates is more difficult. The use of crystal polymorphs to study topological influences upon transfer of excitation energy has been a neglected approach although it may be expected to effectively provide perturbation of lattice parameters without change of the crystal basis.

In the reflection spectra of dye crystals, where there is also a lack of extensive data, characteristic structure which is generally associated with damping is observed. The most distinctive structural feature in the quasimetallic reflection band is a substantial dip in the reflectivity which is observed between the high and low frequency edges of the band. In socalled normal systems this dip is found to be at or near the resonant frequency of the free molecule. ${ }^{4}$ However, there are anomalous cases where the dip is both deep and broad and found to begin more towards the red edge of the reflection band and to be so deep as to divide the band into two apparently separated systems. ${ }^{5}$ This strongly indicates dependence upon the internal field of the crystal. The presence of dissipative processes is also implied and requires refinement.

A model system for the study of these processes is provided by crystal polymorphs of the squarylium dye, 2,4bis-(4-diethylamino-2-hydroxyphenyl) cyclobutadienediylium-1,3,-diolate (HEAPS). Both monoclinic and triclinic crystals have been found and crystal data and spectra from a face of each crystal have been reported. ${ }^{6}$ The two polymorphs exhibit distinctively different optical responses which afford an excellent opportunity for a detailed investigation of the influence of the internal field on the optical structure.

The object of this study is the detailed investigation of the electronic excitations of these strongly coupled crystals. Solution and glass spectra of the free molecule are presented with the crystals' specular reflection spectra and their Kramers-Kronig transforms. Analysis of the data using an expanded molecular polariton approach based on the work of Mahan and Philpott is developed. There is particular fo- 
cus on the determination of the dipole-dipole coupling and the effect of internal electric fields on the reflectivity.

The paper presents in the following section the experimental findings which are followed by assignment of the transitions in the free molecule. These assignments, together with other experimental information on the free molecule and crystal structures, are employed in calculation of the reflection spectra for the various principal directions of the polymorphs in the subsequent section. Effects of various models for the dipole-dipole interaction, coupling of higher states and influence of the internal fields are analyzed in the final section.

\section{EXPERIMENTAL}

The dye, (HEAPS), provided by Exxon Research was used without further purification. It was dissolved in degassed methylene chloride using an ultrasonic cleaner. Crystals were grown in the dark under nitrogen and the two polymorphs normally obtained could be separated by inspection. The monoclinic $\left(P 2_{1} / a, Z=2\right)^{6}$ form grew as large (typically $3 \mathrm{~mm}$ long and $1 \mathrm{~mm}$ on an edge) prismatic crystals with faces $\{001\},\{100\},\{20 \overline{1}\},\{012\}$, and $\{010\}$. Figure 1 shows a typical crystal of the monoclinic polymorph along with the projections onto the $(100)$ and $(20 \overline{1})$ faces.

The triclinic $(P \overline{1}, Z=1)^{6}$ form is a much smaller $(\sim 3 \times 0.1 \mathrm{~mm})$ acicular crystal exhibiting $\{100\},\{1 \overline{1} 0\}$, and $\{001\}$. A typical crystal is shown in Fig. 2 together with the projections onto $(100)$ and $(1 \overline{1} 0)$. In the triclinic system the crystal symmetry does not constrain the principal directions (PD) to lie along any given crystal axis and the polarization of molecular transitions is likely to be the dominant factor controlling these directions.

Near-normal incidence specular reflection spectra were obtained from single crystals of the polymorphs using apparatus similar to that previously described. ${ }^{7}$ Solution spectra were measured on a Cary 14.

\section{MONOCLINIC P2,/a}

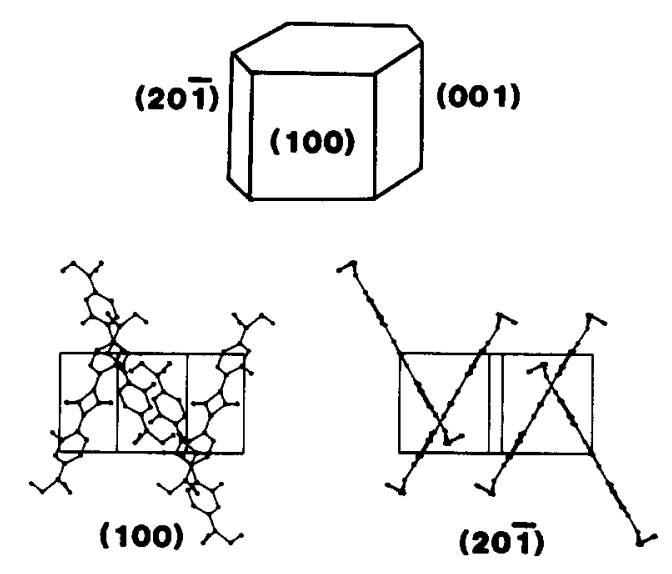

FIG. 1. Crystal morphology and projections for the monoclinic polymorph.

\section{TRICLINIC Pī}

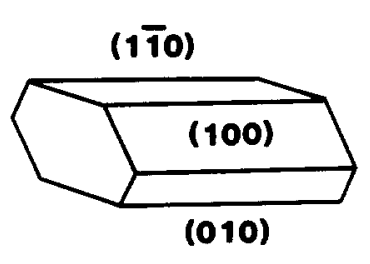

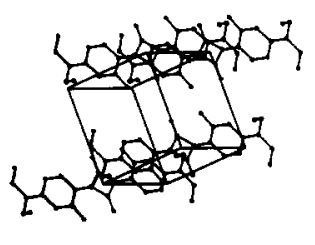

(100)

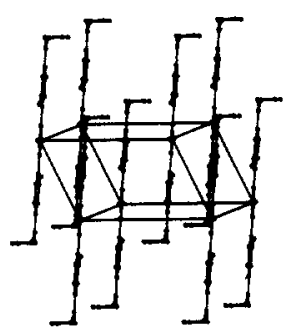

(1ד)
FIG. 2. Crystal morphology and projections for the triclinic polymorph.

\section{RESULTS}

\section{A. Solution and matrix spectroscopy}

The room temperature, and $10^{-6} \mathrm{M}$ spectrum of HEAPS in methylene chloride is shown in Fig. 3. It is comprised of two electronic systems whose transition energies, Franck-Condon factors and dipole lengths are detailed in Table I. In addition a weak transition is observed at 35000 $\mathrm{cm}^{-1}$.

At room temperature, a $10^{-4} \mathrm{M}$ solution showed no evidence of aggregation. Organic glasses at $77 \mathrm{~K}$ in some instances gave a suspension of microcrystals but no true aggregation. ${ }^{8}$ The low solubility of the dye in common glassforming solvents was a severe limitation to formation of aggregates.

The frequency of the lowest energy transition peak is solvent dependent. For a range of solvent dielectric constants from 1.9 to 59 , the peaks shifted $+340 \mathrm{~cm}^{-1}$ from that in methylene chloride. An EPA glass yielded a shift of $-290 \mathrm{~cm}^{-1}$ from the methylene chloride value. In light of the sensitivity of the dye spectra to solvent dielectric properties, quite different solid state spectra are not unexpected for the two polymorphs.

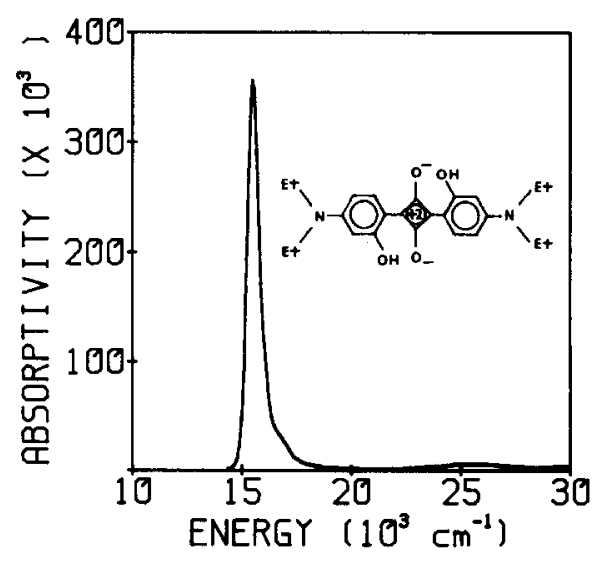

FIG. 3. Solution spectrum of HEAPS at $\approx 300 \mathrm{~K}$ in methylene chloride. 
TABLE I. Features from the solution spectrum of HEAPS in methylene chloride.

\begin{tabular}{cccc}
\hline \hline Transition & $\begin{array}{c}\text { Energy } \\
\left(\mathrm{cm}^{-1}\right)\end{array}$ & $\begin{array}{c}\text { Franck-Condon } \\
\left(\xi^{2}\right)\end{array}$ & $\begin{array}{c}\text { Dipole } \\
\text { length } \\
(\AA)\end{array}$ \\
\hline$S_{1}$ & 15530 & 0.85 & 2.8 \\
& 16670 & 0.13 & \\
$S_{2}$ & 17810 & 0.02 & 0.7 \\
\hline
\end{tabular}

\section{B. Crystal spectroscopy}

\section{Monoclinic}

The absolute reflectivities of the (100) and $(20 \overline{1})$ faces of the monoclinic form are shown in Fig. 4 together with the disposition of the two molecules to the principal directions. The main features are indicated in Table II. The $b$-axis polarized spectrum shows the typical quasimetallic reflection spectrum of a dye, ${ }^{9}$ which is characterized by high reflectivity and a wide, $7500 \mathrm{~cm}^{-1}$ stopping band. This is indicative of strong coupling of excitons and is well within the Simpson and Peterson limit. ${ }^{10} \mathrm{O}$ particular interest is the weak structure to the blue of the stopping band, at $24400 \mathrm{~cm}^{-1}$ since there is some theoretical support for it. ${ }^{5}$

The principal directions for the $B_{u}$ factor group (FG) transitions polarized perpendicular to $b$ are shown for $(100)$ and $(20 \overline{1})$ faces in Fig. 4. For $(100)$ there is substantially diminished intensity. On $(20 \overline{1})$, however, there is high reflectivity most of which is found in the low frequency region and is quite unlike that usually seen in crystals of quasimetallic dyes.

\section{Triclinic}

Dispersion of the principal directions up to $30^{\circ}$ was observed on the (100) face and was found to have little effect on the spectra. On the $(1 \overline{1} 0)$ face, the edgewise orientation of the molecules constrains all in-plane polarized transitions to project parallel to the edge of the projection of the molecular plane. As a result the principal directions remain fixed in the energy region studied and are aligned $9^{\circ}$ and $99^{\circ}$ (designated the max and min directions) off the projected long axis of the molecule.

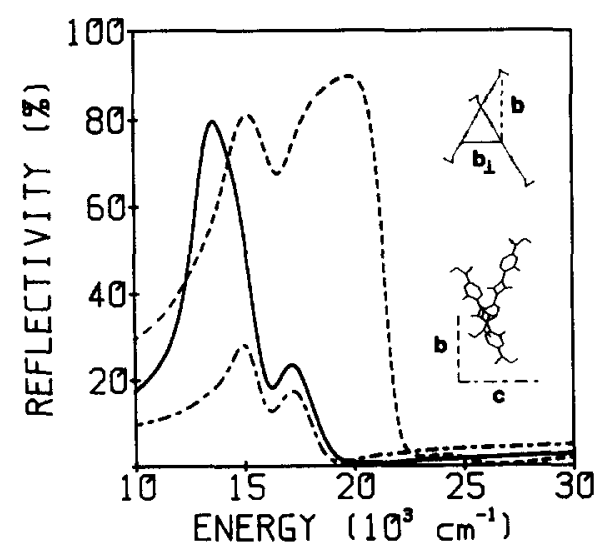

FIG. 4. Absolute reflectivities for the monoclinic (100) and (201) faces.
TABLE II. Spectral features for the HEAPS polymorphs.

\begin{tabular}{|c|c|c|c|c|c|}
\hline \multicolumn{3}{|c|}{ Triclinic } & \multicolumn{3}{|c|}{ Monoclinic } \\
\hline Direction & $\begin{array}{l}\text { Energy } \\
\left(\mathrm{cm}^{-1}\right)\end{array}$ & $\begin{array}{c}\text { Reflectivity } \\
\text { (\%) }\end{array}$ & Direction & $\begin{array}{l}\text { Energy } \\
\left(\mathrm{cm}^{-1}\right)\end{array}$ & $\begin{array}{c}\text { Reflectivity } \\
(\%)\end{array}$ \\
\hline \multirow[t]{5}{*}{ (100) $\max$} & 13400 & 56.5 & $b$ & 15200 & 81 \\
\hline & 15100 & $32^{\mathrm{a}}$ & & 19800 & 90 \\
\hline & 18200 & $26^{n}$ & & 24100 & 2.5 \\
\hline & 21200 & 65 & & & \\
\hline & 24000 & $13^{a}$ & $c$ & 15000 & 28 \\
\hline \multirow[t]{5}{*}{$(1 \overline{1} 0) \max$} & 12800 & 63 & & 17200 & 17.5 \\
\hline & 15100 & $44^{\mathrm{a}}$ & & & \\
\hline & 18000 & $50^{\mathrm{a}}$ & $b_{1}(20 \overline{1})$ & 13600 & 79.5 \\
\hline & 20600 & 65 & & 17200 & 23.5 \\
\hline & 24000 & $12^{\mathrm{a}}$ & & & \\
\hline$(1 \overline{1} 0) \mathrm{min}$ & 13400 & 11 & & & \\
\hline
\end{tabular}

a Shoulder.

The absolute reflectivities and projections for $(100)$ and (110) are shown in Fig. 5 and Table II. The spectra for (100) were measured along the frequency dependent principal directions. The two extremes of the principal directions are indicated in Fig. 5 for (100). The max direction for (100) exhibits a highly distorted quasimetallic reflection spectrum. The apparent stopping band is virtually split in two with a deep minimum at $16700 \mathrm{~cm}^{-1}$, and there is significant structure within the stopping band. This deep dip has only been reported once before in another dye, bis-1,5-(di-

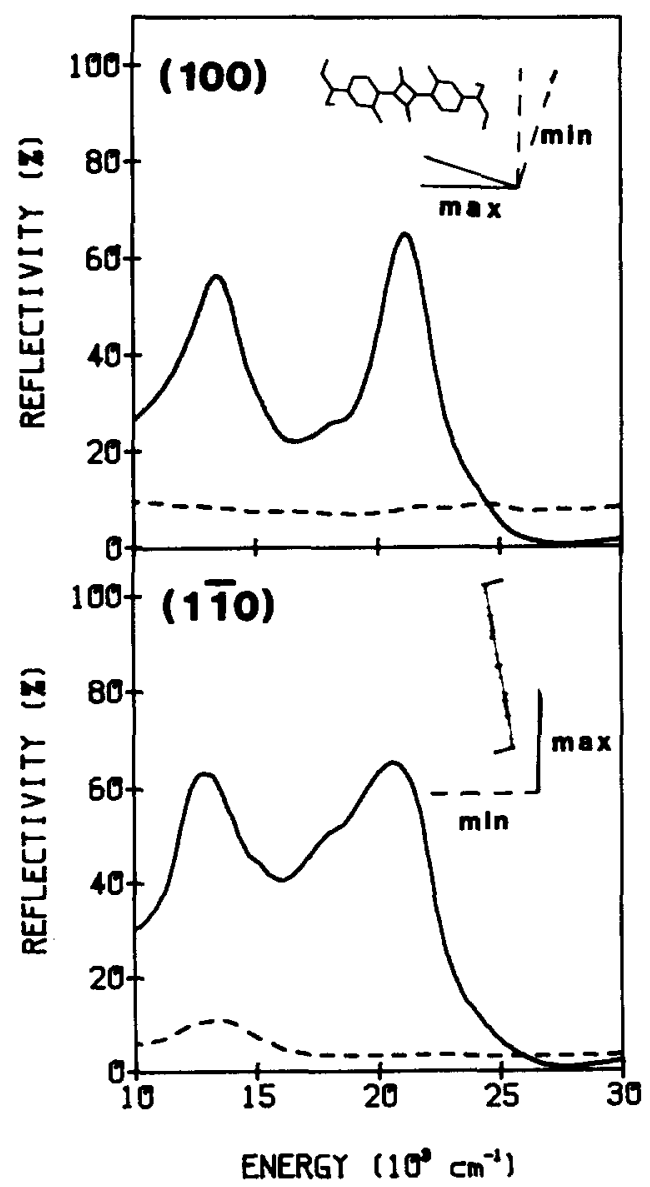

FIG. 5. Absolute reflectivities for the triclinic $(100)$ and $(1 \overline{1} 0)$ faces. 
methylamino) pentamethinium iodide (ADI), and was attributed to strong dissipative processes. ${ }^{5}$ Unlike the $b$ direction in the monoclinic system, there is no structure to the blue of the stopping band although the blue edge shows evidence of a band near $24000 \mathrm{~cm}^{-1}$. The breadth of the stopping band, $10000 \mathrm{~cm}^{-1}$, is greater than that of the monoclinic system and indicates strong coupling. The min direction of the (100) face presents negligible structure.

On $(1 \overline{1} 0)$, the max PD has a less distorted spectrum than seen on (100). The stopping band is $11000 \mathrm{~cm}^{-1}$ wide and there is structure within the band along with distortion of the blue edge near $24000 \mathrm{~cm}^{-1}$. In the min direction there is structure which does not correlate to that in the max direction.

The reflectivity data presented indicate how extremely different the optical responses of the two polymorphs are. Since the progenitor molecular transitions are the same and there are no differences between the two crystals, other than packing, the effects of solid state interactions are undoubtedly determining the radical changes in the spectra.

\section{X-alpha molecular orbltal calculation}

An AIM- $X \alpha-S C F$ calculation similar to that previously described ${ }^{11}$ was made to elucidate the electronic structure of HEAPS. This calculation has proved amenable and efficient for such large systems. The triclinic crystal atomic coordinates were used with a value of 0.8 for alpha. The lowest energy dipole allowed transitions obtained are two nondegenerate singlets $S_{1}$ and $S_{2} . S_{1}$ is from the $\operatorname{HOMO}\left(a_{u}\right)$ to the LUMO $\left(b_{g}\right)$ and $S_{2}$ originates from the penultimately occupied molecular orbital of $a_{u}$ symmetry and terminates with the LUMO. If the long axis is defined as the vector from the center of inversion to the nitrogen, then the polarization of $S_{1}$ is $11^{\circ}$ off the long axis towards the hydroxyl oxygens, while $S_{2}$ is $125^{\circ}$ in the same sense. The hydroxyl oxygens participate inductively in the pi system and this tends to rotate transitions toward the oxygens. ${ }^{12}$

In such limited calculations the calculated transition energies and intensities lack close agreement with experiment, but their relative values are useful. A bulk energy shift of the transition energies to obtain agreement of $S_{1}$ with the lowest energy peak observed in solution places $S_{2}$ at 24300 $\mathrm{cm}^{-1} . S_{1}$ is ten times more intense than $S_{2}$.

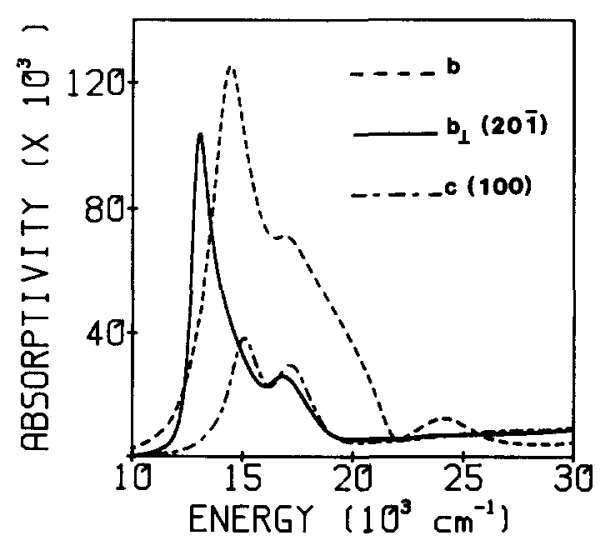

FIG. 6. Kramers-Kronig transforms for the monoclinic crystal.
TABLE III. Kramers-Kronig transform features for the HEAPS polymorphs.

\begin{tabular}{|c|c|c|c|c|c|}
\hline \multicolumn{3}{|c|}{ Triclinic } & \multicolumn{3}{|c|}{ Monoclinic } \\
\hline Direction & $\begin{array}{l}\text { Energy } \\
\left(\mathrm{cm}^{-1}\right)\end{array}$ & $\begin{array}{c}\text { Absorptivity } \\
\left(\ell \mathrm{cm}^{-1} \mathrm{~mol}^{-1}\right)\end{array}$ & Direction & $\begin{array}{l}\text { Energy } \\
\left(\mathrm{cm}^{-1}\right)\end{array}$ & $\begin{array}{c}\text { Absorptivity } \\
\left(\ell \mathrm{cm}^{-1} \mathrm{~mol}^{-1}\right.\end{array}$ \\
\hline$(100) \max$ & $\begin{array}{l}13300 \\
14800 \\
18400 \\
20400 \\
24000\end{array}$ & $\begin{array}{l}7.54 \times 10^{4} \\
4.38 \times 10^{4} \\
4.37 \times 10^{4} \\
7.46 \times 10^{4} \\
2.11 \times 10^{4}\end{array}$ & $b$ & $\begin{array}{l}14400 \\
16900 \\
24100\end{array}$ & $\begin{array}{l}1.25 \times 10^{5} \\
7.11 \times 10^{4} \\
1.23 \times 10^{4} \\
3.82 \times 10^{4}\end{array}$ \\
\hline$(1 \overline{1} 0) \max$ & $\begin{array}{l}12800 \\
15100 \\
17900 \\
18900 \\
24000\end{array}$ & $\begin{array}{l}9.36 \times 10^{4} \\
5.85 \times 10^{4} \mathrm{a} \\
7.14 \times 10^{4} \\
7.14 \times 10^{4} \\
2.21 \times 10^{4} \mathrm{a}\end{array}$ & $b_{1}(20 \overline{1})$ & $\begin{array}{l}13100 \\
16800\end{array}$ & $\begin{array}{l}2.96 \times 10^{4} \\
1.04 \times 10^{5} \\
2.60 \times 10^{4}\end{array}$ \\
\hline$(1 \overline{1} 0) \min$ & 14400 & $1.34 \times 10^{4}$ & & & \\
\hline
\end{tabular}

"Shoulder.

\section{Kramers-Kronig transforms}

\section{Monoclinic}

The resultant absorption curves obtained by KramersKronig transform of the reflection spectra for the monoclinic polymorph are depicted in Fig. 6 and Table III. The similarity of the $b$ axis and $b$-perpendicular spectra for the (201) face in contrast to their quite different appearance in their reflection spectra demonstrates the interpretive problem of arguing solely from reflection spectra. The peaks at 14400 and $16900 \mathrm{~cm}^{-1}$ in the b-axis spectrum are the $0-0$ and $0-1$ vibronic components of $S_{1}$, and the $24100 \mathrm{~cm}^{-1}$ peak is associated with $S_{2}$.

For the perpendicular $b$ direction on the (201) face, the $0-0$ component of $S_{1}$ occurs at $13100 \mathrm{~cm}^{-1}$ and the $0-1$ at $16800 \mathrm{~cm}^{-1}$. The asymmetry of the $0-0$ component is evident in the transform and indicates a large exciton shift for that component. The Davydov splittings for the $0-0$ and $0-1$ components of $S_{1}$ on (201) and (100) are in Table IV. Dipole lengths calculated from integrated intensities are given in Table V for $S_{1}$.

The dichroic ratio $(\beta)$ will be defined as the ratio of intensity in the $b$-axis polarized spectrum to that in the perpendicular $b$ direction for the monoclinic faces and max to min for the triclinic faces. The dichroic ratios for transition moments obtained from the $X$-alpha calculation are shown in Table $V$ along with the experimental dichroic ratios for the first singlet transition. The dichroic ratio for the $(20 \overline{1})$ face is a very slowly varying function of angle in the region of interest, so the experimental value of 4.3 for the (100) face was

TABLE IV. Davydov splittings $\left(\mathrm{cm}^{-1}\right)$ for $S_{1}$ in monoclinic polymorph.

\begin{tabular}{ccrrr}
\hline & Component & Experimental & Long axis & $11^{\circ}$ \\
\hline$(20 \overline{1})$ & 0 & 1300 & 1820 & 1931 \\
& 1 & 100 & 24 & 25 \\
$(100)$ & 0 & -700 & -682 & 1242 \\
& 1 & -300 & -21 & 9 \\
\hline \hline
\end{tabular}

Transition moment used for calculated spectra in Fig. 9.

${ }^{b} X$-alpha transition moment for best fit listed in Table VI. 
TABLE V. Dichroic ratios for various transition moments along with crystal dipole lengths

\begin{tabular}{|c|c|c|c|c|c|c|}
\hline & \multicolumn{5}{|c|}{ Dichroic ratio $(\beta)$} & \multirow{2}{*}{$\begin{array}{l}\text { dipole } \\
\quad \text { lengths }(\AA ̊ \AA)^{\mathrm{b}}\end{array}$} \\
\hline & $\theta=11$ & $\theta=6$ & $\theta=135$ & $\exp \left(S_{1}\right)^{\mathrm{a}}$ & $\theta=125$ & \\
\hline $\begin{array}{r}\text { Monoclinic }(100) \\
(20 \overline{1})\end{array}$ & $\begin{array}{l}2.7 \\
3.2\end{array}$ & $\begin{array}{l}4.2 \\
2.7\end{array}$ & $\begin{array}{l}4.3 \\
3.1\end{array}$ & $\begin{array}{l}4.3 \\
2.1\end{array}$ & $\begin{array}{l}3.3 \\
1.3\end{array}$ & $\begin{array}{l}2.6(b), 2.8(c) \\
3.0\left(b_{1}\right)\end{array}$ \\
\hline $\begin{array}{ll}\text { Triclinic } & (100) \\
& (1 \overline{1} 0)\end{array}$ & $\begin{array}{r}520 \\
33\end{array}$ & $\begin{array}{l}50 \\
36\end{array}$ & $\begin{array}{l}0.5 \\
199\end{array}$ & $\begin{array}{l}\text { large } \\
12.3\end{array}$ & $\begin{array}{c}0.3 \\
>999\end{array}$ & $\begin{array}{l}2.4(\max ) \\
2.5(\max )\end{array}$ \\
\hline
\end{tabular}

Obtained from Kramers-Kronig transform integrated intensities.

${ }^{b}$ Calculated for the directions indicated in parentheses with $a 6^{\circ}$ transition moment.

used to make the polarization assignment. This resulted in possible transition moments at $6^{\circ}$ or $135^{\circ}$ to the long axis. A transition moment $6^{\circ}$ from the long axis yields dichroic ratios (Table V) for the (100) and (201) faces in good agreement with the experimental values. For the second singlet, $\beta$ is indeterminate because the ill-defined structure in the perpendicular $b$ direction in the $S_{2}$ energy region prevents definition of the band shape. $\beta>1$ for the two monoclinic faces, however, is consistent with the $X$-alpha assignment of $125^{\circ}$ for $S_{2}$.

Use of the real part $\epsilon_{1}$ of the complex dielectric constant obtained from the transforms permits definition of the range of the stopping band. In the $b$ polarization direction corresponding to the $A_{u}$ factor group, $\epsilon_{1}$ becomes negative in the 14000 to $21500 \mathrm{~cm}^{-1}$ energy region. On the $(20 \overline{1})$ face, the $B_{u}$ factor group direction is found to have negative values of $\epsilon_{1}$ from 12900 to $17800 \mathrm{~cm}^{-1}$.

\section{Triclinic}

The transformed absorption spectra for the triclinic crystal are shown in Fig. 7 and in Table III. For $(100)$ the real part of the dielectric function is found to be negative from 13200 to 15400 and from 19600 to $23400 \mathrm{~cm}^{-1}$. Two regions with $\epsilon_{1}<1$ are also found on the (010) face of TCNQ ${ }^{\circ}{ }^{13}$ although not to the extent seen with HEAPS. The min direction for $(100)$ shows no significant structure.

In the max direction for (110), $\epsilon_{1}$ falls below zero from 12800 to $23200 \mathrm{~cm}^{-1}$. The min direction has a single broad peak.

Theoretical $\beta$ 's were also determined for the triclinic faces and are listed along with experimental $S_{1}$ values in Table V. For $(100)$ the ratio is extremely large since there is little identifiable structure in the min polarization direction. For neither the theoretically calculated transition moment $\left(11^{\circ}\right)$ nor the $6^{\circ}$ moment found by examining the monoclinic transforms does the experimental dichroic ratio for the triclinic (110) agree. That experimental value includes the 14400 $\mathrm{cm}^{-1}$ peak in the min direction. Exclusion of that peak makes $\beta$ larger and puts it in general agreement with the $6^{\circ}$ or $11^{\circ}$ moment assignment. It is useful to define an alternative dichroic ratio $\left(\beta^{\prime}\right)$ as $I_{\max }(1 \overline{1} 0) / I_{\max }(100)$ which has an experimenal value of 1.26 whereas the theoretical values for a $6^{\circ}$ and $11^{\circ}$ transition moment are 1.31 and 1.29. In addition to the previous arguments, the assignment of a moment at $135^{\circ}$ would be ruled out by consideration of the $(100)$ triclinic face where that assignment would result in $\beta=0.5$. Thus, the polarization of the first singlet consistent with both polymorphs is $6^{\circ}$. Errors inherent in determining an experimental dichroic ratio exist but even a $20 \%$ error in the $\beta$ value for the (100) monoclinic face would result in an angular change of only $\pm 3^{\circ}$ from the present assignment. For the oriented gas model used here, $S_{1}$ may be taken as polarized slightly off the long axis in the direction of the hydroxyl oxygens. The agreement with the oriented gas model is reflected in the dipole lengths determined from integrated intensities (Table V).

In Table V, the $X$-alpha polarization of $S_{2}\left(125^{\circ}\right)$, on the triclinic faces leads to $\beta$ 's of 0.3 and $>999$ for the $(100)$ and (110) faces, respectively. An experimental value for $S_{2}$ is difficult to ascertain, but it is clear that $\beta$ should be significantly

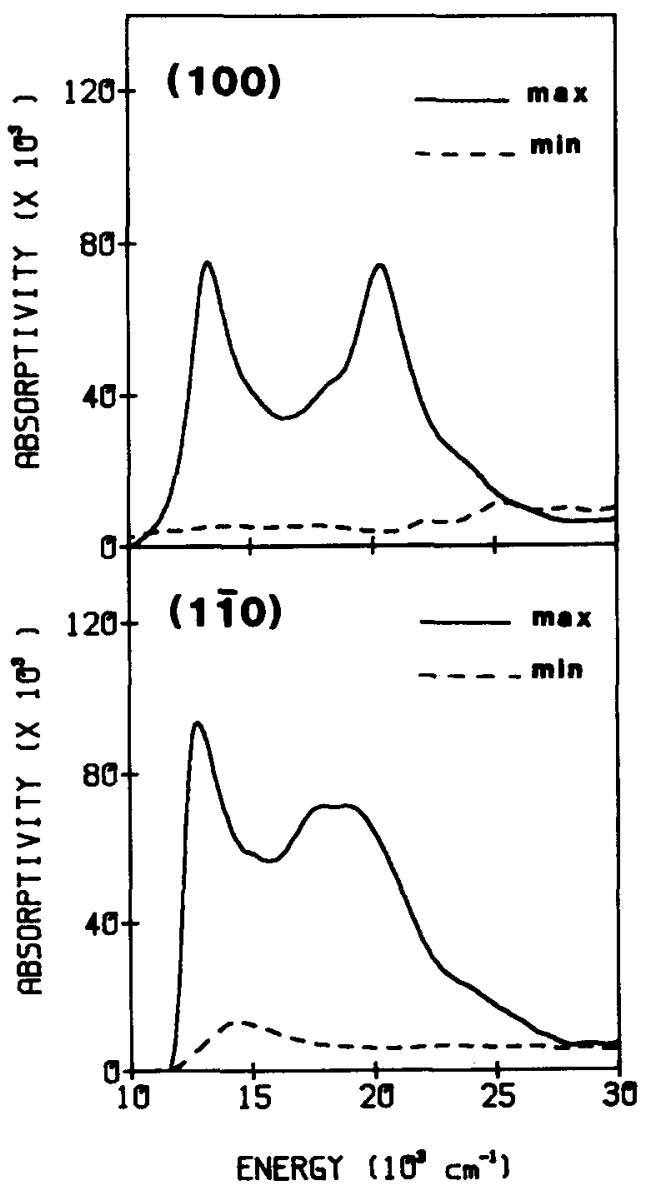

FIG. 7. Kramers-Kronig transforms for the triclinic crystal. 
greater than 1 for both faces. A polarization for $S_{2}$ consistent with all four faces is that it is essentially $6^{\circ}$ off the long axis.

\section{DISCUSSION}

\section{A. Charge transfer band}

The $14400 \mathrm{~cm}^{-1}$ transition in the min direction of the (110) triclinic face, which was excluded from the dichroic ratio calculation of $S_{1}$, is assigned as an intermolecular charge transfer transition. From the packing of the molecules an ideal arrangement exists for an intermolecular transition. This is most clearly seen from the ( $1 \overline{1} 0)$ projection. The molecules are as close as $3.35 \AA$ and overlayed so that the nitrogen of one molecule almost lies over the center of the four member ring of the second. As Tanaka has pointed out, such a staircase arrangement is quite favorable for a charge resonance interaction in dye crystals. ${ }^{14}$ In the min direction of the ( $1 \overline{1} 0)$ face the normal to the molecular plane ( $z$ axis) has a large projection on the principal direction. An intermolecular transition polarized perpendicular to the plane of the molecule should not be observable on (100) since there the $z$ axis is $108^{\circ}$ from the min principal direction. The min direction of the (100) face reveals no significant structure.

Further support for an intermolecular charge transfer transition lies in consideration of the shape of the band in the (110) min direction. The band does not mirror the structure seen in the max direction as would be expected if it were a projection of the same transition. In addition, a charge transfer transition is suggested by the location of the principal directions in the (110) face. The long axis transitions are completely transverse to the wave vector of the incident light. If only in-plane transitions were considered in this orientation, the principal directions should lie along the projected edge (long axis) of the molecule and perpendicular to it. The principal directions are found, however, to be rotated $9^{\circ}$ and $99^{\circ}$ off the long axis of the molecule. This can be explained if there is an out-of-plane transition in the same energy region as the in-plane transition. The principal directions would reflect the polarization of each transition and the effect of a weak out-of-plane transition would thereby be to rotate slightly the principal directions off the projections of the in-plane transitions.

It is conceivable that the band is due to a vibronically allowed out-of-plane component of $S_{1}$. This would be consistent with the location of the peak and the principal directions. However, the band shape does not correlate with the structure in the max direction and it shows no hint of the congested structure associated with such transitions. The $14400 \mathrm{~cm}^{-1}$ band may then be assigned as a charge transfer band.

\section{B. Molecular polariton theory}

\section{Single state}

In strongly coupled systems a theoretical description amounts to solving for the polariton dispersion curves wherein stationary states of the coupled exciton and photon fields must be explicitly considered. This coupling is introduced by using the retarded electromagnetic field as the exciting field. Although a complete theoretical description would necessitate a multiparticle theory, a single-particle approach developed by Philpott ${ }^{15}$ and based on earlier work by Mahan ${ }^{16}$ can be illuminating.

It was shown in an earlier paper ${ }^{6}$ that the application of a single state molecular polariton theory to the $(100)$ faces of the polymorphs failed to describe the experimental spectra. Similar results obtain for the calculated spectra of the (201) and $(1 \overline{1} 0)$ faces. The relevant equations have only two adjustable parameters: the background dielectric function $\epsilon_{0}$ and vibronic damping constants $\Gamma_{u}$. Other qualities are either measured or calculated. Of the calculated quantities, only the analytic lattice sum admits of different computational approaches. In the previous study where agreement was not obtained, the point dipole approximation was used in calculating the lattice sums. Although there are dye crystals in which it has proven to be a good approximation, here it is suspect because of the size of the transition dipoles involved. Since this approximate polariton theory considers only one state and allows for no explicit coupling to higher electronic transitions, effects arising from such coupling can not be discounted.

It has been known for some time that the point dipole approximation is not always appropriate nor valid. ${ }^{17}$ At small intermolecular distances relative to the size of the molecules, it is a poor approximation of the total resonance interaction. ${ }^{18}$ Two models will be explored, the extended dipole and the charge density.

In the extended dipole model, ${ }^{19}$ two centers of opposite charge in the molecule located at a distance $d$ apart define the charge distribution. For two extended dipoles, the calculation involves the interaction of four charges in a pairwise fashion. This would be expected to better reflect the actual extent of these rather sizeable transition dipoles.

The dipoles were oriented in the direction of the $S_{1}$ theoretically calculated transition moment, and the distance between the charges was varied. The charges, symmetrically located about the inversion center of the molecule, were scaled to yield solution transition dipole strengths. For the monoclinic case a value of $d=10 \AA$ gave good results. The analytic lattice sum for the interaction of molecules at equivalent positions $\left(4 \pi / V_{0}\right) t_{1 r, 1}(0)$ (numbers specify the summation site, letters the transition: $r=S_{1}$ and $V_{0}$ is the volume of the unit cell) is $-295 \mathrm{~cm}^{-1} / \AA^{2}$, and for the inequivalent $\operatorname{sum}\left(4 \pi / V_{0}\right) t_{1 r, 2 r}(0)$ is $-77 \mathrm{~cm}^{-1} / \AA^{2}$. Therefore, the factor group sums are -218 and $-372 \mathrm{~cm}^{-1} / \AA^{2}$ for $A_{u}$ and $B_{u}$ factor groups. Table I contains the spectroscopic information for $S_{1}$ used in calculating the reflection spectra and Table VI lists the parameters for the calculations. The calculated spectra for the monoclinic system using the extended dipole sums are shown in Fig. 8. The spectra are in good agreement with experiment for such a simple model and are certainly superior to those obtained using point dipole sums. For the $b$ direction, the bandwidth of the spectrum 7700 $\mathrm{cm}^{-1}$ is only $200 \mathrm{~cm}^{-1}$ wider than that of the experimental one. The perpendicular $b(20 \overline{1})$ spectrum is blue shifted 800 $\mathrm{cm}^{-1}$, and the main band is broader than the experimental one. There also is a $700 \mathrm{~cm}^{-1}$ red shift in the perpendicular $b$ (100) calculated spectrum relative to the experimental one.

The calculated spectra for the triclinic crystal in Fig. 8, use a value of $d=6 \AA$ resulting in a lattice sum of -808 
TABLE VI. Parameters for calculated reflection spectra.

\begin{tabular}{|c|c|c|c|c|}
\hline & Direction & $\begin{array}{c}\text { Factor } \\
\text { group sum }\end{array}$ & $\begin{array}{l}\text { Damping } \\
\text { constant }^{\mathrm{b}}\end{array}$ & $\begin{array}{c}\text { Background } \\
\text { dielectric constant }\end{array}$ \\
\hline \multicolumn{5}{|l|}{ Fig. 8} \\
\hline Monoclinic & $\begin{array}{l}b \\
b_{1}(20 \overline{1}) \\
c(100)\end{array}$ & $\begin{array}{l}-218 \\
-372 \\
-372\end{array}$ & $\begin{array}{l}800,999,100 \\
400,800,80 \\
2000,600,100\end{array}$ & $\begin{array}{l}1.8 \\
1.5 \\
1.5\end{array}$ \\
\hline Triclinic & $\begin{array}{l}(1 \overline{1} 0) \max \\
(100) \max \end{array}$ & $\begin{array}{l}-808 \\
-808\end{array}$ & $\begin{array}{l}999,999,999 \\
999,999,999\end{array}$ & $\begin{array}{l}1.5 \\
1.5\end{array}$ \\
\hline \multicolumn{5}{|l|}{ Fig. 9} \\
\hline Monoclinic & $\begin{array}{l}b \\
b_{\perp}(20 \overline{1}) \\
c(100) \\
(1 \overline{1} 0) \max \\
(100) \max \end{array}$ & $\begin{array}{l}-200 \\
-400 \\
-400 \\
-350 \\
-700\end{array}$ & $\begin{array}{l}800,999,100 \\
500,500,100 \\
999,300,50 \\
999,999,999 \\
999,999,999\end{array}$ & $\begin{array}{l}1.9 \\
1.5 \\
1.5 \\
2.0 \\
1.5\end{array}$ \\
\hline $\begin{array}{l}\text { Best fit } 11^{\circ} \\
\text { transition momen } \\
\text { Monoclinic }\end{array}$ & $\begin{array}{l}b \\
b_{\perp}(20 \overline{1}) \\
c(100)\end{array}$ & $\begin{array}{l}-200 \\
-456 \\
-456\end{array}$ & $\begin{array}{l}800,999,1000 \\
500,400,50 \\
2000,600,80\end{array}$ & $\begin{array}{l}1.8 \\
1.5 \\
1.5\end{array}$ \\
\hline
\end{tabular}

$\left(4 \pi / V_{0}\right)\left[t_{1 r, 1 r}(0) \pm t_{1 r, 2 r}(0)\right]$ in $\mathrm{cm}^{-1 / \AA^{2}}$.

${ }^{\mathrm{b}} \Gamma_{u}^{1}$ in $\mathrm{cm}^{-1}$.

$\mathrm{cm}^{-1} / \AA^{2}$. Here there is less success in matching the experimental results and the calculated band for the $(1 \overline{1} 0)$ max direction only roughly approximates the blue edge of the stopping band. This somewhat ad hoc placement of the dipole in the extended dipole model is not particularly satisfy-

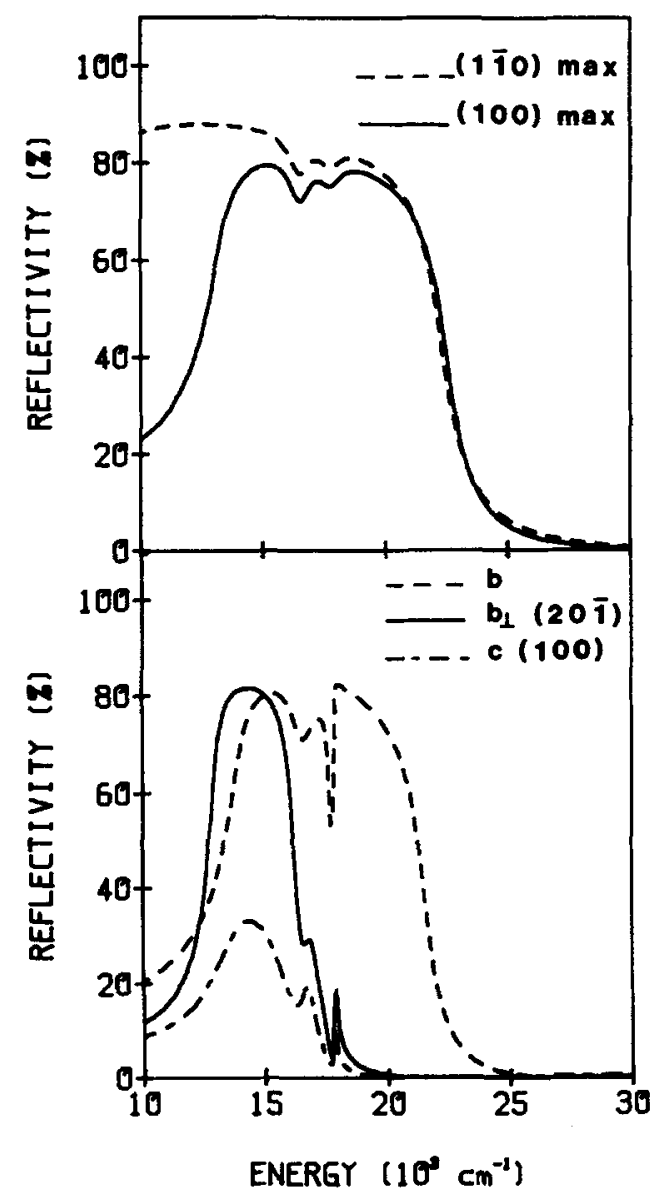

FIG. 8. Calculated spectra for the triclinic (upper) and monoclinic (lower) crystals using extended dipole lattice sums. ing nor is its one dimensional nature. A model that better reflects the geometry of the charge distribution of the molecule should be considered and may aid in calculating the spectra for the triclinic polymorph.

The atomic monopole or point charge model ${ }^{17}$ allows one to incorporate into the lattice sum calculations the geometry and symmetry of the charge distribution of the molecule. The model may be expected to better describe the actual charge distributions and electrostatic interactions of the molecules in the crystal. In this model, the resonance interaction energy is calculated from the interaction of point charges located at each atomic center. As in the extended dipole method the $11^{\circ}$ transition moment was used with a direct Lorentz-Lorenz sum to calculate the dipole-dipole interactions. ${ }^{20}$ The radius of the summation sphere varied from 80 to $110 \AA$ depending upon the particular crystal system. For the monoclinic case, the $\left(4 \pi / V_{0}\right) t_{1 r, 1 r}(0)$ and $(4 \pi /$ $\left.V_{0}\right) t_{1,, 2 r}(0)$ sums are -418 and $-38 \mathrm{~cm}^{-1} / \AA^{2}$, yielding $A_{u}$ and $B_{u}$ factor group sums of -380 and $-456 \mathrm{~cm}^{-1}$, $\AA^{2}$, whereas $\left(4 \pi / V_{0}\right) t_{1 r, 1 r}(0)$ is $-278 \mathrm{~cm}^{-1} / \AA^{2}$ for the triclinic crystal. The calculated spectra represent no significant improvement over the extended dipole results, except that the perpendicular $b(20 \overline{1})$ direction matched the experimental curve better. The experimental triclinic spectra were once again poorly reproduced.

In an effort to test the limit of the single state theory used, the analytic lattice sums were used as freely adjustable parameters in fitting the calculated spectra. The position of the calculated band, its band shape and the calculated exciton energies in relation to the experimental results were the criteria used in the fitting procedure. The macroscopic fields were calculated and not treated as adjustable. The long axis transition moment assignment was used in fitting the calculated curves. The $\epsilon_{0}$ term incorporates the effects of higher energy transitions on the $S_{1}$ transition. It was observed that increasing the value of $\epsilon_{0}$ affected the high energy edges of the bands by moving them towards lower energies. The vari- 


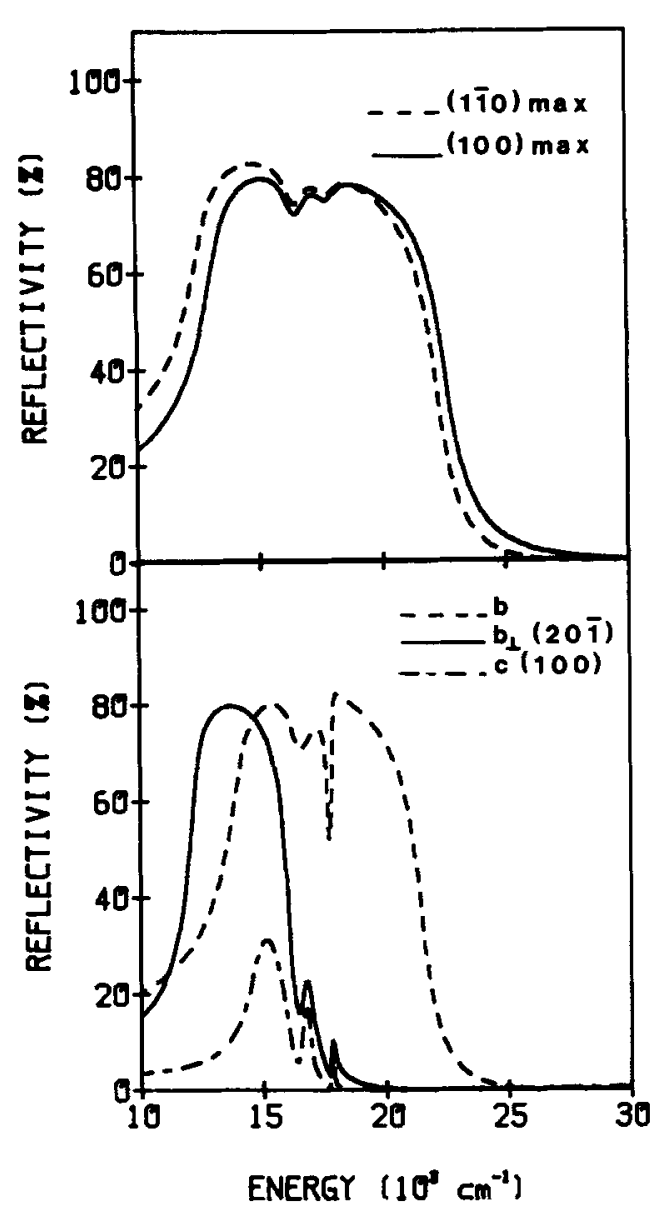

FIG. 9. Calculated spectra using fitted sums and a long axis transition moment for the triclinic (upper) and monoclinic (lower) crystals.

ation of the analytic lattice sums was manifest in the position of the red edge of the bands. Increasingly negative values of the FG sums moved the red edge of the band to lower energies. No reasonable fit was obtained without the FG sums as shown in Table VI.

The resultant fitted spectra are shown in Fig. 9 and the corresponding parameters for the plots are listed in Table VI. In the triclinic case the best agreement only allowed definition of the edges of the stopping band. The single state theory used cannot describe the multiparticle region where there is much structure. In the monoclinic system the stopping bandwidths as well as the band shapes reasonably agree with the measured spectra. The large variance in the perpendicular $b$ polarized directions is explained in terms of directional dispersion. This sensitivity to $\mathbf{k}$ was brought to light in a slightly different manner when the Davydov splittings for the monoclinic case were compared using the best fit with a long axis transition moment and the theoretically calculated one of $11^{\circ}$. These transition moments can be thought of as lower and upper bounds on the experimental $6^{\circ}$ moment.

The best fit with the $X$-alpha transition moment used the parameters listed in Table VI in the calculation of the spectra. A tabulation of the Davydov splittings for the two transition moments and the experimental results from the transforms is shown in Table IV. A very large discrepancy is obtained for the splittings for the perpendicular $b(100)$ direction; in fact, the direction of the splitting using the $X$-alpha
TABLE VII. Factor group macroscopic lattice terms calculated for different transition moments. ${ }^{\mathrm{a}}$

\begin{tabular}{llcc}
\hline \hline & Direction & Long axis $^{\mathrm{b}}$ & $11^{\circ \mathrm{b}}$ \\
\hline Monoclinic & $b$ & 0 & 0 \\
& $b_{1}(20 \overline{1})$ & 6 & 142 \\
& $\mathrm{c}(100)$ & 854 & 354 \\
Triclinic & $(1 \overline{10}) \max$ & & 121 \\
& $(100) \max$ & & 779 \\
\hline
\end{tabular}

$\overline{\mathrm{a}}\left(4 \pi / V_{0}\right)(\hat{\mu} \cdot \hat{\mathbf{k}})$ in $\mathrm{cm}^{-1} / \AA^{2}$.

${ }^{b}$ Transition moment.

transition moment is reversed from that observed. The reason can be ascertained by examining Table VII where the two transition moment choices are compared. There is such a large projection of either transition moment in both factor group directions on the $(20 \overline{1})$ face, that there is little difference between the two. But in the perpendicular $b(100)$ direction the small difference in orientation between the transition moments results in a large difference in the macroscopic field $(\hat{\boldsymbol{\mu}} \cdot \hat{\mathbf{k}})$ terms. It is those terms which determine the values of the Coulombic excitons (see Ref. 15) and thereby the splittings. This demonstrates the dependence of the crystal spectra, particularly in the strong coupling regime, on the macroscopic field.

In Table VIII comparison is made between the best fit spectra and those using point dipole, point charge and extended dipole analytic lattice sums. The FG sums and $\epsilon_{0}$ values used are shown along with the exciton energies which define the stopping band and the bandwidth. These energies and bandwidths are compared to the values from the transforms. For the sake of comparison, the $X$-alpha transition moment is used for all calculations. In the perpendicular $b$ (201) direction of the monoclinic polymorph the best fitted sum was the point charge lattice sum which resulted in a 50 $\mathrm{cm}^{-1}$ shift between the calculated band and the experimental one. In all cases, the improvement in going from point dipole approximation to the point charge is evident. In some instances the extended dipole gave good results but the uncertainty in locating that dipole makes the point charge model the preferred method for these dye systems. In addition there is less manipulation of the point charge sums wherein only the charges are scaled to yield free molecule oscillator strengths. In the extended dipole method not only are the charges scaled but the positions of the charges are adjustable.

A more physical interpretation of the analytic lattice sums is instructive in the comparison of the different methods of calculating internal fields for the polymorphs. The analytic lattice sums used for the polymorphs in the fitting procedure and those calculated by the point dipole and point charge models are shown in Table IX. Examination of the crystal packing and the point dipole sums for equivalent molecules reveals that the localized nature of the point dipole model fails to account for a side by side positive interaction of the large transition dipoles. The use of the point charge model which incorporates a more realistic charge distribution results in less negative lattice sums which approach the best fit values. However, in the triclinic polymorph the point charge model overcompensates for the spatial exten- 
TABLE VIII. Exciton energies ${ }^{\mathrm{a}}$ and stopping bandwidths $\left(\mathrm{cm}^{-1}\right)$.

\begin{tabular}{|c|c|c|c|c|c|}
\hline & Best fit & Ext. dipole ${ }^{b}$ & Pt. charge ${ }^{b}$ & Pt. dipole ${ }^{b}$ & Experimental \\
\hline \multicolumn{6}{|l|}{$\begin{array}{c}\text { Monoclinic } \\
b\end{array}$} \\
\hline FG Sum $\left(\mathrm{cm}^{-1} / \AA^{2}\right)$ & -200 & -218 & -380 & -1091 & \\
\hline$\epsilon_{0}$ & 1.8 & 1.8 & 1.6 & 1.6 & \\
\hline Coulombic & 14001 & 13838 & 12356 & $<256$ & 14000 \\
\hline $\mathbf{k}=0$ polariton & 21563 & 21536 & 21393 & 17845 & 21500 \\
\hline Bandwidth & 7652 & 7698 & 9037 & $>16736$ & 7500 \\
\hline $\begin{array}{c}b_{1}(20 \overline{1}) \\
\text { FG Sum }\left(\mathrm{cm}^{-1} / \AA^{2}\right)\end{array}$ & -456 & -372 & -456 & -1088 & \\
\hline$\epsilon_{0}$ & 1.5 & 1.5 & 1.5 & 1.5 & \\
\hline Coulombic & 12070 & 12891 & 12070 & $<256$ & 12900 \\
\hline $\mathbf{k}=0$ polariton & 17821 & 17857 & 17821 & 17779 & 17800 \\
\hline Bandwidth & 5751 & 4966 & 5751 & $>17523$ & 4900 \\
\hline \multicolumn{6}{|l|}{$\begin{array}{l}\text { Triclinic } \\
\qquad(1 \overline{1} 0) \max \end{array}$} \\
\hline FG Sum $\left(\mathrm{cm}^{-1} / \AA^{2}\right)$ & -450 & -808 & -278 & -1328 & \\
\hline$\epsilon_{0}$ & 1.8 & 1.5 & 2.0 & 2.0 & \\
\hline Coulombic & 12329 & 8255 & 13843 & $<256$ & 12800 \\
\hline $\mathbf{k}=0$ polariton & 22528 & 22219 & 22645 & 17810 & 23200 \\
\hline Bandwidth & 10199 & 13964 & 8802 & $>17554$ & 10400 \\
\hline$(100) \max$ & & & & & \\
\hline FG Sum $\left(\mathrm{cm}^{-1} / \AA^{2}\right)$ & -800 & -808 & -278 & -1328 & \\
\hline$\epsilon_{0}$ & 1.5 & 1.5 & 2.0 & 2.0 & \\
\hline Coulombic & 13285 & 13215 & 16127 & 4064 & $13200 / 19600$ \\
\hline $\mathbf{k}=0$ polariton & 22555 & 22510 & 22861 & 17819 & $15400 / 23400$ \\
\hline Bandwidth & 9270 & 9295 & 6734 & 13755 & 10200 \\
\hline
\end{tabular}

a Exciton energies shown that define the stopping bands. Factor group lattice sums calculated for an $11^{\circ}$ transition moment.

${ }^{b}$ Charge distribution model used in calculated spectra.

sion of the charge distribution and results in a less negative lattice sum than that found by the fitting procedure. Perfect agreement is not expected considering that the charge densities are calculated by approximate methods. In the monoclinic form, the inequivalent sum calculated by the point charge model is in closest agreement to the best fit value.

The examination of the lattice sums from the fitting procedure affords the best estimate of the internal fields of the polymorphs. In the triclinic polymorph the equivalent sum is more negative than that of the monoclinic form. This is particularly evident for the triclinic (100) face which exhibits a stopping band split in two with a shape reminiscent of the joint density of states function of a one dimensional crystal.

Another difference between the two polymorphs is manifested in the blue edges of the stopping bands in the $b$ direction for the monoclinic case and the max directions in the triclinic. In the first a small peak outside the stopping

TABLE IX. Lattice sums ${ }^{\mathrm{a}}\left(\mathrm{cm}^{\left.-1 / \AA^{2}\right)}\right.$ used in single state theory.

\begin{tabular}{lrrl}
\hline \hline & Point dipole $^{\mathrm{b}}$ & Point charge $^{\mathrm{b}}$ & Best fit \\
\hline Monoclinic & & & \\
$t_{1 r, 1 r}(0)$ & 2 & -418 & -328 \\
$t_{1,, 2 r}(0)$ & & & -128 \\
Triclinic & -1328 & -278 & $-800(100)$ \\
$t_{1 r, 1 r}(0)$ & & & $-450(1 \overline{1} 0)$
\end{tabular}

"Calculated for an $11^{\circ}$ transition moment, $\left(4 \pi / V_{0}\right)$ omitted for compactness.

${ }^{b}$ Charge distribution model used in calculated spectra. band is observed to higher energies, and in the other a band is apparently convoluted into the stopping band. The theory of Fanconi et al. ${ }^{5}$ does predict structure outside the stopping band toward higher energies. An alternative is that in both cases the effect of the coupling $S_{1}$ and $S_{2}$ is operant. This would not be expected to be large because of the low intensity of $S_{2}$ but could be mitigated by its like polarization and close proximity to $S_{1}$. Inclusion of some exciton-exciton coupling may also better define the triclinic spectra. In an effort to explore this possibility, a polariton theory using two states was sought that incorporates explicit coupling between electronic states.

\section{Two state}

Mahan's formulation of polariton theory includes an explicit coupling of two electronic states. ${ }^{16}$ Using these equations and recasting them into a form compatible with calculations employed previously allows more convenient comparison to the single state form.

The equations of motion of a dipole in the presence of an electromagnetic field are the basis for a derivation of the crystal dielectric function. The unit cell is considered to have two translationally inequivalent molecules. The free molecule oscillator strength may be written

$$
f_{u}^{j}=2 m \omega_{u} u_{j} \xi_{u}^{2} / \hbar e^{2} .
$$

Then the energy dependent polarizability of a state is

$$
4 \pi \alpha_{j}(E)=\left(4 \pi u_{j}^{2} / V_{0}\right) A_{j}(E),
$$

where 


$$
A_{j}(E)=\sum_{u} 2 e_{u} \xi_{u}^{2} / e_{u}^{2}-E^{2},
$$

$m$ is the mass of an electron, $\omega_{u}$ is the $u$ th free molecule excitation energy in $\mathrm{cm}^{-1}, u_{j}$ is the dipole length of the $j$ th transition, $\xi_{u}^{2}$ is the Franck-Condon factor for the $u$ th free molecule vibronic transition with excitation energy $e_{u}, E$ is the energy, and $V_{0}$ is the volume of the unit cell.

The point dipole interaction energy terms can be recast as

$$
T_{r, s}=\left(V_{0} / 4 \pi u_{r} u_{s}\right)\left[I_{\alpha r, \alpha s}(\mathbf{k}) \pm I_{\alpha r, \beta s}(\mathbf{k})\right],
$$

where the $r, s$ specify the electronic transition and $\alpha, \beta$ is the site of the molecule in the unit cell. The \pm refers to the two factor groups. The interaction energy can be broken into two parts. The first is an analytic function of $k$, the exciton wave vector, and the other, the so-called macroscopic term, is a nonanalytic function of $\mathbf{k}$. For optical frequencies, the analytic term can be replaced by its value at $\mathbf{k}=0$. Then

$$
I_{\alpha r, \beta s}(\mathbf{k})=\left(4 \pi u_{r} u_{s} / V_{0}\right)\left[t_{\alpha r, \beta s}(0)+\left(\hat{\boldsymbol{\mu}}_{\alpha r} \cdot \hat{\mathbf{k}}\right)\left(\hat{\boldsymbol{\mu}}_{\beta s} \cdot \hat{\mathbf{k}}\right)\right],
$$

where $\hat{\mu}_{\alpha r}$ is the transition moment for transition $r$ on molecule $\alpha$.

Following the treatment of Philpott, ${ }^{15}$ a background dielectric constant is included to account for the effects of the higher electronic states not explicity considered. This modifies the interaction energy, but does not affect the analytic term since it is dominated by close dipole pair interactions. Thus

$$
I_{\alpha r, \beta s}(\mathbf{k})=u_{r} u_{s}\left[i_{\alpha r, \beta s}(0)+\left(4 \pi / V_{0} \epsilon_{0}\right)\left(\hat{\boldsymbol{\mu}}_{\alpha r} \cdot \hat{\mathbf{k}}\right)\right]
$$

where $i_{\alpha r, \beta s}(0)=\left(4 \pi / V_{0}\right) t_{\alpha r, \beta s}(0)$.

The resultant form of the dielectric function is

$$
\begin{aligned}
n^{2}= & \epsilon_{0}+2\left(4 \pi / V_{0} \Delta\right)\left(u _ { r } ^ { 2 } A _ { r } ( E ) \left\{1+A_{s}(E)\right.\right. \\
& \left.\times\left[I_{1 s, 1 s}(\mathbf{k}) \pm I_{1 s, 2 s}(\mathbf{k})\right]\right\}\left(\hat{\mathbf{e}}_{\lambda} \cdot \hat{\boldsymbol{\mu}}_{1 r}\right)^{2}+u_{s}^{2} A_{s}(E) \\
& \times\left\{1+A_{r}(E)\left[I_{1 r, 1 r}(\mathbf{k}) \pm I_{1 r, 2 r}(\mathbf{k})\right]\right\}\left(\hat{\mathbf{e}}_{\lambda} \cdot \hat{\boldsymbol{\mu}}_{1 s}\right)^{2} \\
& -2\left(\hat{\mathbf{e}}_{\lambda} \cdot \hat{\boldsymbol{\mu}}_{1 r}\right)\left(\hat{\mathbf{e}}_{\lambda} \cdot \hat{\boldsymbol{\mu}}_{1 s}\right) u_{r} u_{s} A_{r}(E) A_{s}(E) \\
& \left.\times\left[I_{1 r, 1 s}(\mathbf{k}) \pm I_{1 r, 2 s}(\mathbf{k})\right]\right)
\end{aligned}
$$

where

$$
\begin{aligned}
\Delta= & \left\{1+A_{r}(E)\left[I_{1 r, 1 r}(\mathbf{k}) \pm I_{1 r, 2 r}(\mathbf{k})\right]\right\}\left\{1+A_{s}(E)\left[I_{1 s, 1 s}(\mathbf{k})\right.\right. \\
& \left.\left. \pm I_{1 s, 2 s}(\mathbf{k})\right]\right\}-A_{r}(E) A_{s}(E)\left[I_{1 r, 1 s}(\mathbf{k}) \pm I_{1 r, 2 s}(\mathbf{k})\right]^{2} .
\end{aligned}
$$

The $\hat{\mathbf{e}}_{\lambda}$ is the light polarization for the factor group of interest and the other quantities are defined as before. Some phenomenological damping is introduced to account for relaxation channels of the vibronic excitons due to decay into the

\begin{tabular}{|c|c|c|c|c|}
\hline & \multicolumn{2}{|c|}{ Monoclinic } & \multicolumn{2}{|c|}{ Triclinic } \\
\hline & Pt. charge ${ }^{b}$ & Pt. dipole ${ }^{b}$ & Pt. charge ${ }^{b}$ & Pt. dipole \\
\hline \multirow[t]{2}{*}{$\begin{array}{l}i_{1 r, 1 r}(0) \\
i_{1,2 r}(0) \\
i_{1 s, 1 s}(0) \\
i_{1 s, 2 s}(0) \\
i_{1 r, 1 s}(0) \\
i_{1 r, 2 s}(0)\end{array}$} & $\begin{array}{r}-418 \\
-38 \\
-418 \\
-38 \\
-418 \\
-38\end{array}$ & $\begin{array}{r}-1089 \\
2 \\
-1089 \\
2 \\
-1089 \\
2 \\
\text { Mon }\end{array}$ & $\begin{array}{l}-278 \\
-278 \\
-278 \\
\text { clinic }\end{array}$ & $\begin{array}{l}-1328 \\
-1328 \\
-1328\end{array}$ \\
\hline & $\begin{array}{r}\quad A_{u} \text { fac } \\
\text { Pt. charge }\end{array}$ & $\begin{array}{l}\text { group } \\
\text { Pt. dipole }\end{array}$ & $\begin{array}{c}\quad B_{u} \text { fact } \\
\text { Pt. charge }\end{array}$ & $\begin{array}{l}\text { group } \\
\text { Pt. dipole }\end{array}$ \\
\hline $\begin{array}{l}\operatorname{Sum}(r) \\
\operatorname{Sum}(s) \\
\operatorname{Sum}(r s)\end{array}$ & $\begin{array}{l}-456 \\
-456 \\
-456\end{array}$ & $\begin{array}{l}-1091 \\
-1091 \\
-1091\end{array}$ & $\begin{array}{l}-380 \\
-380 \\
-380\end{array}$ & $\begin{array}{l}-1087 \\
-1087 \\
-1087\end{array}$ \\
\hline
\end{tabular}
multiparticle regions, scattering by lattice phonons and other lattice energy dissipation mechanisms. This gives

$$
\boldsymbol{A}_{j}(E)=\sum_{\mathrm{u}} 2 e_{u} \xi_{u}^{2} / e_{u}^{2}-E^{2}-i E \Gamma_{u}^{j},
$$

where $\Gamma_{u}^{1}$ and $\Gamma_{u}^{2}$ are the damping constants for the $u$ th vibronic levels of the first and second transitions. By considering only one electronic transition, Eq. (2) simplifies to the single state form used earlier. ${ }^{6}$

Both point charge and point dipole lattice sums were
TABLE X. Lattice sums ${ }^{\mathrm{a}}\left(\mathrm{cm}^{-1} / \AA^{2}\right)$ used in two state theory.

${ }^{\circ}$ Calculated for an $11^{\circ}$ transition moment.

${ }^{\mathrm{b}}$ Charge distribution model used in calculated spectra.

used in this extension of Mahan's theory. A problem arises in that the charge distributions from the $X$-alpha calculation needed to calculate point charge sums give an incorrect transition moment polarization for $S_{2}$. The $S_{2}$ transition is experimentally polarized parallel to the $S_{1}$ transition so the same transition charge distributions were used for the two singlets. However, the distributions were scaled to yield the dipole strengths appropriate to the transition. In Table $\mathrm{X}$, the different analytic lattice sums calculated by using the point charge and point dipole models for the polymorphs are presented. For the monoclinic case, the different factor group sums are defined as follows: $\operatorname{sum}(r)=i_{1 r, 1 r}(0) \pm i_{1 r, 2 r}(0), \operatorname{sum}(s)=i_{1 s, 1 s}(0) \pm i_{1 s, 2 s}(0) \quad$ and $\operatorname{sum}(r s)=i_{1 r, 1 s}(0) \pm i_{1 r, 2 s}(0)$. The triclinic form has no factor group splitting, and therefore, the sums used are the individual lattice sums. Notice that in the single state theory, the $\operatorname{sum}(r)$ values were used. The fact that the polarizations of $S_{1}$ and $S_{2}$ are taken as identical makes $\operatorname{sum}(r)=$ sum $(s)=\operatorname{sum}(r s)$. As before, the point dipole sums in this calculation gave very poor results, and the spectra calculated using the point charge sums do not give a good description of the actual spectra except for the perpendicular $b$ (201) direction.

A fitting procedure adjusting the analytic lattice sums was employed. Three lattice sum parameters are available for adjustment, one for each transition and one representing a coupling between the two transitions. Variance of the $\operatorname{sum}(r)$ and $\operatorname{sum}(s)$ terms leads to a shift of their respective bands with increasingly negative sums moving the bands to lower energies. As before, the $\epsilon_{0}$ term affects the higher energy edge of the bands with an increase in $\epsilon_{0}$ displacing the edge to lower energies. Since the sum(rs) term is a measure of the coupling between the singlets, its adjustment varied the peak height of $S_{2}$ and the stopping bandwidth of $S_{1}$. Increasingly negative values of sum(rs) lead to greater coupling. Both edges of $S_{1}$ are affected and shift in opposite directions to increase the bandwidth at the expense of $S_{2}$ which diminished in intensity. Although there are several adjustable parameters, no resonable reflection curve can be calculated without a value of $\operatorname{sum}(r s)$ in the vicinity of the FG sum 


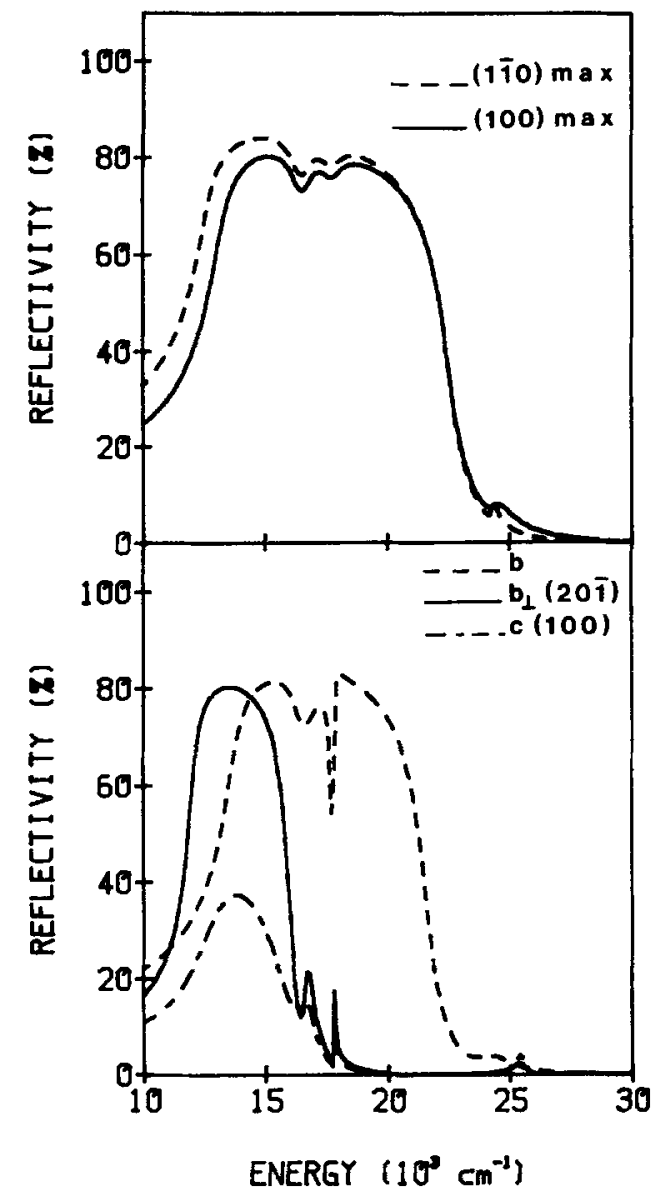

FIG. 10. Calculated spectra using fitted sums and including $S_{1}$ and $S_{2}$ for the triclinic (upper) and monoclinic (lower) polymorphs.

values used in the single state form of the polariton theory. Plots for the monoclinic and triclinic polymorphs are shown in Fig. 10 and Table XI lists the parameters for each plot.

The adapted theory of Mahan describes the monoclinic $b$ direction fairly well. The best fit for this direction reproduces the stopping band width along with the general band shape. The structure near $24000 \mathrm{~cm}^{-1}$ matches that of the experimental spectrum. In all of the calculated spectra for this direction, the shape of the multiparticle region was not well described. After a dip in reflectivity at $17800 \mathrm{~cm}^{-1}$, the calculated spectra rise much more rapidly and peak at lower energy than the experimental spectra. The fitted value for $\operatorname{sum}(r)$ (see Table XI) is in better agreement with the point charge calculation of sum $(r)$ than the point dipole one. (Table $\mathrm{X})$. In contrast, best fit values for $\operatorname{sum}(s)$ and $\operatorname{sum}(r s)$ are closer to the point dipole sums.

In the perpendicular to $b(20 \overline{1})$ and (100) directions the best fitted lattice sum values were once again identical to those calculated by the point charge model. Weak structure is observed in both directions at $25400 \mathrm{~cm}^{-1}$ which correlates with the slow rise in reflectivity from 20000 to 30000 $\mathrm{cm}^{-1}$ in the experimental spectra. The main peak in the calculated (100) spectrum is displaced slightly from the experimental peak.

For the max directions of the triclinic (100) and (110) faces, the best that could be achieved with the fitted sums was to match the bandwidths of the experimental curves. Higher values of damping constants did not lower the calculated stopping band region to match the experiment; rather it washed out the little structure present in that region. For both the $(100)$ and $(1 \overline{1} 0)$ faces, $S_{2}$ appears to be responsible for the structure near $24000 \mathrm{~cm}^{-1}$ within the stopping bands. The transition is not strong enough to perturb the middle of the stopping band which indicates that other types of interactions contribute to that structure. As seen in the monoclinic case the $\operatorname{sum}(r)$ point charge values were much closer to the best fit ones than were the point dipole sums and the reverse is true for the sum(s) and sum(rs) terms (see Tables $\mathrm{X}$ and XI). It appears that in both polymorphs the point charge model overcompensates for the spatial extension of the transition charge distribution for $S_{2}$.

The difference between the high energy edges of the stopping band in the monoclinic $b$ direction and the max directions of the triclinic polymorph is found to be due to a difference in the coupling of the two singlet transitions. The $\operatorname{sum}(r s)$ values for the best fits are much more negative for the

TABLE XI. Parameters for calculated spectra using two states.

\begin{tabular}{|c|c|c|c|c|c|c|c|c|c|}
\hline & \multicolumn{5}{|c|}{ Figure 10} & \multicolumn{2}{|c|}{ Figure 11} & \multicolumn{2}{|c|}{ Figure 12} \\
\hline & $b$ & $b_{\perp}(20 \overline{1})$ & $c(100)$ & $(1 \overline{1} 0) \max$ & $(100) \max$ & $b$ & $b_{1}(20 \overline{1})$ & $(1 \overline{1} 0) \max$ & $(100) \max$ \\
\hline $\begin{array}{l}\text { Lattice sums } \\
\text { Sum(r) } \\
\text { Sum }(s) \\
\text { Sum(r) }\end{array}$ & $\begin{array}{r}-200 \\
-4000 \\
-800\end{array}$ & $\begin{array}{l}-456 \\
-456 \\
-456\end{array}$ & $\begin{array}{l}-456 \\
-456 \\
-456\end{array}$ & $\begin{array}{r}-300 \\
-4000 \\
-1700\end{array}$ & $\begin{array}{r}-700 \\
-4000 \\
-1700\end{array}$ & $\begin{array}{r}-200 \\
-4000 \\
-800\end{array}$ & $\begin{array}{l}-456 \\
-456 \\
-456\end{array}$ & $\begin{array}{r}-300 \\
-4000 \\
-1500\end{array}$ & $\begin{array}{r}-700 \\
-4000 \\
-1700\end{array}$ \\
\hline $\begin{array}{c}\text { Damping cons } \\
\Gamma_{1}^{1} \\
\Gamma_{2}^{1} \\
\Gamma_{3}^{1} \\
\Gamma_{1}^{2}\end{array}$ & $\begin{array}{r}800 \\
999 \\
100 \\
2000\end{array}$ & $\begin{array}{r}500 \\
400 \\
50 \\
600\end{array}$ & $\begin{array}{r}2000 \\
600 \\
80 \\
400\end{array}$ & $\begin{array}{l}999 \\
999 \\
999 \\
200\end{array}$ & $\begin{array}{l}999 \\
999 \\
999 \\
999\end{array}$ & $\begin{array}{c}800,0.5,0^{\mathrm{c}} \\
999,0.1,0^{c} \\
500,0.1,0^{\mathrm{c}} \\
2000,100,0^{\mathrm{c}}\end{array}$ & $\begin{array}{r}500 \\
400 \\
50 \\
600\end{array}$ & $\begin{array}{c}999,0.1,300^{c} \\
999,0.1,300^{c} \\
999,0.1,300^{c} \\
200,100,0^{c}\end{array}$ & $\begin{array}{c}999,0.1,300^{c} \\
999,0.1,300^{c} \\
999,0.1,300^{c} \\
999,100,0^{c}\end{array}$ \\
\hline $\begin{array}{l}\text { Background } \\
\text { dielectric }\left(\epsilon_{0}\right)\end{array}$ & 1.8 & 1.5 & 1.5 & 2.0 & 1.6 & 1.8 & 1.5 & 2.0 & 1.6 \\
\hline
\end{tabular}

${ }^{a}$ Units in $\mathrm{cm}^{-1} / \AA^{2}$.

${ }^{b}$ Superscripts label the singlets and subscripts the vibronic levels in each. Units in $\mathrm{cm}^{-1}$

${ }^{c}$ The three numbers are those needed to define each $\Gamma_{u}^{j}(E)$ for damping according to Eq. (4). 
triclinic stopping bands than the monoclinic. This trend is indicative of a larger coupling between the singlets in the triclinic crystal, however, it must be remembered that the $\operatorname{sum}(r s)$ is just the analytic part of the resonance interaction term $I_{\alpha r, \beta s}(\mathbf{k})$. For the $b$ direction in the monoclinic crystal, the $r s$ interaction term is $I_{1 r, 1 s}(\mathbf{k})-I_{1 r, 2 s}(\mathbf{k})$, with the resultant being $i_{1 r, 1 s}(0)-i_{1 r, 2 s}(0)$, since the macroscopic terms cancel each other out. In the triclinic case, the macroscopic terms remain (Table VII). In both the triclinic faces the total $r s$ lattice sum is more negative than the monoclinic $b$ direction, reflecting the greater coupling between the singlet transitions.

The explicit inclusion of a second singlet in the polariton calculations was successful at explaining the structure in the region of the high energy edge of the stopping bands. However, the multiparticle regions remain poorly defined. In particular, the location of the second peak in the $b$-axis reflection spectrum is incorrect and the structure for the stopping bands of the triclinic polymorph is in poor agreement. In the calculations presented, a frequency independent damping has been associated with the vibronic components. The damping term accounts for losses due to various lattice relaxation mechanisms. It is reasonable to expect these losses to be frequency dependent. ${ }^{21}$ Several damping functions were explored ${ }^{22}$ including an exponential form expected for relaxation through phonon channels ${ }^{23}$ but the most successful one was a Gaussian of the form

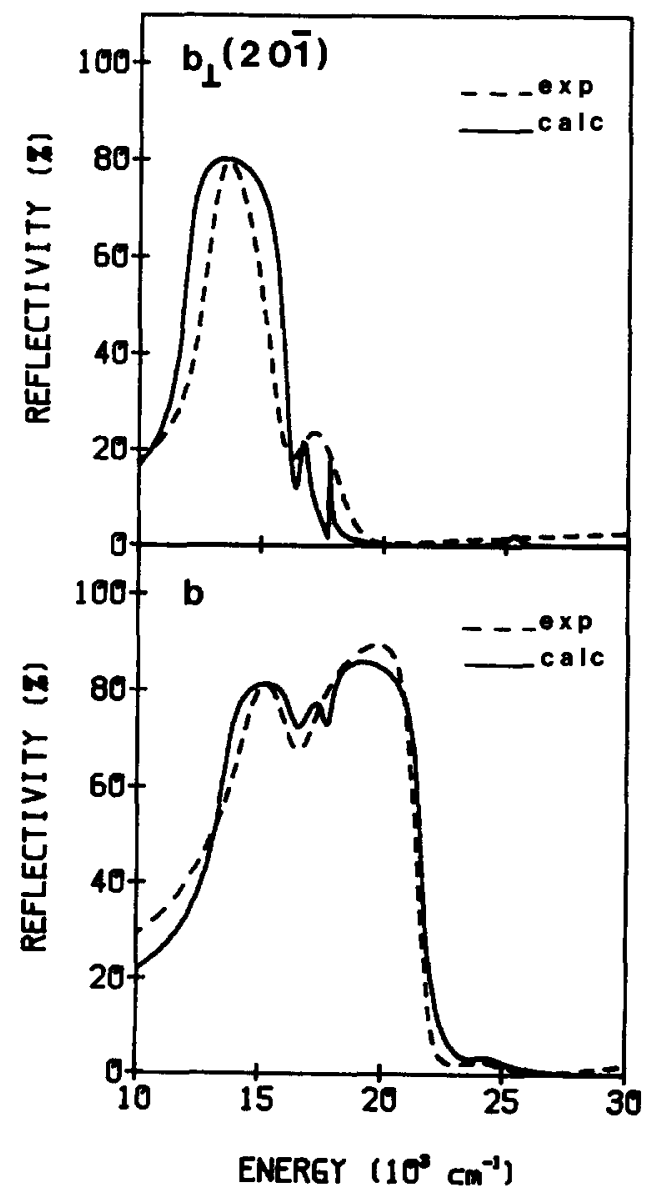

FIG. 11. Calculated spectra for the monoclinic $b$ axis (includes a Gaussian damping function) and the perpendicular $b(20 \overline{1})$ direction are compared to the experimental spectra. Both $S_{1}$ and $S_{2}$ are considered.

$$
\Gamma_{u}^{j}=A \exp \left[-\left(E-e_{u} / e_{u} B\right)^{2}\right]+C,
$$

where $A$ was on the order of the frequency independent damping previously used.

The calculated spectrum using this function, along with the experimental spectrum, is shown for the monoclinic $b$ direction in Fig. 11. Parameters are in Table XI. The effect of the frequency dependent damping for $S_{1}$ is to place the peak in the multiparticle region further to the blue and be in better agreement with the experimental curve than that obtained using frequency independent damping. $S_{2}$ structure was reproduced without using frequency dependent damping. The agreement of the calculated spectrum with the experimental one is now quite good.

For the perpendicular $b$ (201) direction no improvement was obtained by including frequency dependent damping in the calculation. In Fig. 11 the best calculated spectrum is shown. The direct comparison of the calculated spectrum with the experimental one supports the conjecture that the observed peak at $17200 \mathrm{~cm}^{-1}$ is the result of two peaks. A similar broadening was observed in the perpendicular $b(100)$ direction.

The Gaussian damping function for $S_{1}$ only, was used in the calculation of the spectra for the max polarization directions of the triclinic faces (Fig. 12). The agreement of the calculated spectrum with the experimental one for the (11i0) face is remarkable. The structure within the stopping band is

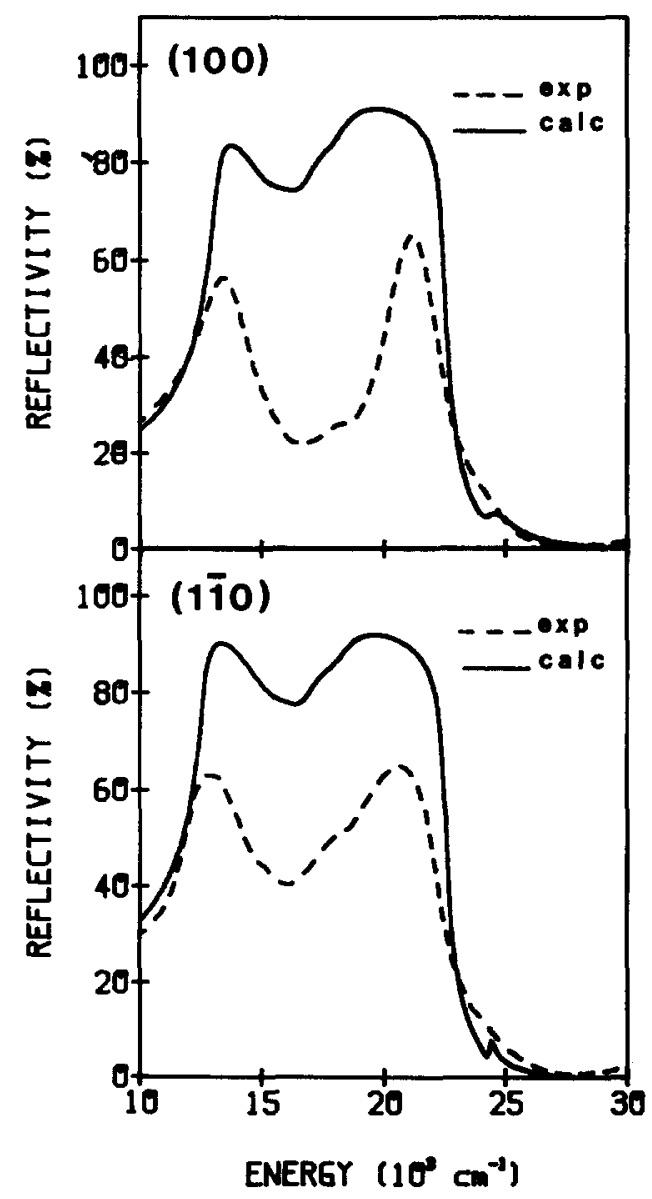

FIG. 12. Calculated spectra considering $S_{1}$ and $S_{2}$ and using a Gaussian damping function for the triclinic polymorph are compared to the experimental spectra. 


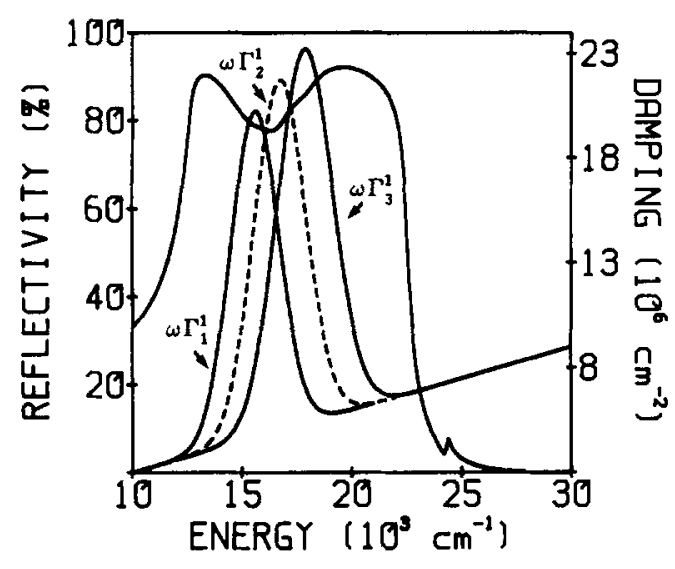

FIG. 13. The Gaussian damping functions $\omega \Gamma^{1}$ are shown alongside the calculated spectrum for the (110) max direction as in Fig. 12.

reproduced and only the magnitude of the reflectivity is in disagreement with the experimental curve. Notwithstanding its large separation from the $12800 \mathrm{~cm}^{-1}$ peak, the structure at 15100 and $18000 \mathrm{~cm}^{-1}$ is shown to be vibronic and illustrates the difficulty of extracting such information from strongly coupled systems. The calculation of the (100) max direction was not as successful and the well separated peaks observed experimentally can not be reproduced.

There is no strong theoretical reason to expect the damping to be described by such a Gaussian function. Given the complexity and lack of knowledge of damping phenomena, other functions are just as valid and in some instances an exponential function worked well. To understand this damping better, it is useful to know the energy region spanned by the frequency dependent damping functions. In Fig. 13 the calculated spectra for the $(1 \overline{1} 0)$ max direction is shown along with plots of the damping functions for each vibronic component of $S_{1} ; \Gamma_{1}^{1}, \Gamma_{2}^{1}, \Gamma_{3}^{1}$ multiplied by the polariton energy in $\mathrm{cm}^{-1}$. The damping is introduced phenomenologically in the polarizability as a product of $\Gamma^{j}{ }_{u}$ and energy [see Eq. (3)]. The resultant damping functions $\omega \Gamma^{j}{ }_{u}$ are Gaussians superimposed on a linearly increasing background, with half-widths on the order of $4000 \mathrm{~cm}^{-1}$. Appropriately, the structure in the stopping band is correlated with the regions in which the damping functions vary greatly from the background value. The low and high energy edges of the stopping band can be reproduced using a frequency independent value of $\Gamma_{u}^{1}$. This not unexpected result is found for the other spectra as well.

With the introduction of frequency dependent damping, the molecular polariton theory has been extended to its limit. The band shapes and positions of the experimental reflection spectra for the monoclinic polymorph and the (110) max direction of the triclinic crystal were described well. There are still some problems describing the triclinic spectra in that different analytic lattice sums were necessary to fit the $(1 \overline{1} 0)$ and $(100)$ faces.

\section{CONCLUSION}

An extensive study of the specular reflection spectra of the monoclinic and triclinic polymorphs of a squarylium dye (HEAPS) has revealed the complexity of interactions in these strongly coupled systems. Elucidation of the effect of the internal fields upon the optical response must necessarily be based upon the properties of the constituent molecules. This requirement is met through assumption of an oriented gas model for the assignment of the first two singlet molecular transitions. These are polarized $6^{\circ}$ from the long axis of the molecule with the first comprised of three vibrational components. The observation of an out-of-plane band in the triclinic crystal can be attributed to an intermolecular charge transfer transition. The assumption that the electronic transitions are those of the free molecule is subject to doubt in systems such as these where tightly packed, highly polarizable molecules are subject to large fields. However, it is unlikely that the differences can be too great and it is diffcult to devise a reasonably tractable alternative. The subsequent agreement of the calculated spectra based upon these assignments lends support to the assumption.

The radically different reflection spectra of the two polymorphs, which are representative of the extremes previously observed for quasimetallically reflecting crystals, can be treated using the theory of molecular polaritons. A consequence of this investigation is that the lattice sum calculations for these large, extended systems with high oscillator strength transitions should not be executed using the point dipole approximation. The failure of the point dipole approximation for the calculation of the reflection spectra leads to the use of point charges to calculate the lattice interaction. While the adoption of a more realistic charge distribution produces reasonable agreement of bandwidth and energies, structure on the blue edge of the reflection band must be replicated by including coupling to a weak but close lying singlet state. This has little additional effect on the calculated curve save for the production of the weak structure at the high energy edge which is seen in the experimental reflection spectra. A transition of greater intensity would undoubtably produce greater effect. Directional dispersion accounts for much of the observed change of shape of the reflection bands in these systems. In fact, the monoclinic spectra are reasonably well described without further addition to the theory. The triclinic system, however is little improved by accounting for directional dispersion.

The major problem of the reflection spectra of dye crystals which display structure similar to that found in the triclinic crystal wherein the band is nearly split in two is not solved by the above modifications. Since the region of the band involved is associated with relaxation processes, the crystal damping was examined. The use of constant damping provides little improvement and frequency dependent damping is found necessary for a description of the crystal relaxation processes. Since the damping considered was included in the crystal dielectric function, it should account for decay channels associated with the lattice. For decay through phonon channels, an exponential dependence on the frequency would be expected. While such a response provided some improvement in the monoclinic spectra it was not otherwise effective. However, quite good results were obtained using a combined Gaussian and linear dependence on the frequency for each of the first singlet vibronic levels. The effectiveness of this form of damping is of particular 
interest since the usual exponential expression for phonon relaxation processes was not nearly as effective. A purely linear dependence was also of slight use.

A more realistic physical approach to calculating the reflection spectra is certainly of value. The agreement with experiment is actually surprisingly good for such a limited model. The general moelcular polariton approach is an effective one to understanding these strongly coupled systems.

The lack of aggregation spectra for the dye in solution is unfortunate since it prevents comparison of such spectra to those of the dye crystal polymorphs. However, the crystal packings of the polymorphs are of the types expected for aggregation, and to this degree, provide insight on the influence of structure on spectra. Determination of the desired spectra-structure correlation for both aggregate and crystal must await a system wherein comparison of the crystal polymorph spectra to that of the aggregate can be achieved. Nevertheless, this approach, which is the first to approach understanding of aggregation by use of polymorphism, provides a method of approach for the determination of the structure of dye aggregates.

The observation of the quasimetallic stopping band in both factor group directions on the (201) face of the monoclinic crystal admits of supporting surface exciton polariton states. This is the first such molecular crystal wherein such excitations can be supported in more than one direction. The understanding of these surface excitations will be furthered by the ability to study the two dimensional dispersion of these surface excitations. Work in this area is now being pursued.

An understanding of the optical and electronic responses of dyes is necessary for many commercial uses. The propensity of dyes to form polymorphs suggests a systematic approach to structure-spectra correlation which should be useful in understanding dye aggregation as well as other interactions in a strongly coupled lattice.

\section{ACKNOWLEDGMENTS}

The dye was provided by D. L. Morel, formerly of Exxon Research. The point charge lattice calculations were made utilizing a program kindly provided by C. L. Zinsser and S. C. Neely. The authors also wish to thank A. P. Marchetti, R. H. Young, and E. I. P. Walker who have investigated the spectra of the monoclinic form of HEAPS and provided us with their unpublished data.

'E. E. Jelley, Nature 138, 1009 (1936); G. R. Bird, K. S. Norland, A. E. Rosenoff, and H. B. Michaud, Photogr. Sci. Eng. 12, 196 (1968); D. L. Smith. ibid., 18, 309 (1974); S. Makio, N. Kanamaru, and J. Tanaka, Bull. Chem. Soc. Jpn. 53, 3120 (1980).

${ }^{2}$ M. R. Philpott, Adv. Chem. Phys. 23, 227 (1973); R. R. Pennelly and C. J. Eckhardt, Chem. Phys. Lett. 9, 572 (1971).

${ }^{3}$ A. P. Marchetti, C. B. Salzberg, and E. I. P. Walker, Photogr. Sci. Eng. 20, 107 (1976).

${ }^{4}$ B. G. Anex and W. T. Simpson, Rev. Mod. Phys. 32, 466 (1960).

${ }^{5}$ B. G. Fanconi, G. A. Gerhold, and W. T. Simpson, Mol. Cryst. Liquid Cryst. 6, 41 (1969).

${ }^{6} \mathrm{M}$. Tristani-Kendra, C. J. Eckhardt, J. Bernstein and E. Goldstein, Chem. Phys. Lett. 98, 57 (1983). The dye in this paper was erroneously labeled HBAPS instead of HEAPS.

${ }^{7}$ R. R. Pennelly, Ph.D. thesis, University of Nebraska, 1972.

${ }^{8}$ Theory of the Photographic Process, 4th ed., edited by T. H. James (Macmillan, New York, 1977).

${ }^{9}$ R. R. Pennelly and C. J. Eckhardt, Chem. Phys. 12, 89 (1976).

${ }^{10}$ W. T. Simpson and D. L. Peterson, J. Chem. Phys. 26, 588 (1957).

"J. Tylicki, R. J. Hood, G. A. Gallup, and C. J. Eckhardt, J. Chem. Phys. 66, 3745 (1977).

${ }^{12}$ J. N. Murrell in The Theory of the Electronic Spectra of Organic Molecules (Chapman and Hall, London, 1971), Chap. 9.

${ }^{13}$ M. R. Philpott, P. M. Grant, K. Syassen, and J-M. Turlet, J. Chem. Phys. 67, 4229 (1977).

${ }^{14} J$. Tanaka, M. Tanaka, M. Hayakawa, Bull. Chem. Soc. Jpn. 53, 3109 (1980).

${ }^{15}$ M. R. Philpott, J. Chem. Phys. 54, 2120 (1971).

${ }^{16}$ G. D. Mahan, J. Chem. Phys. 41, 2930 (1964).

${ }^{17}$ F. London, J. Phys. Chem. 46, 305 (1942).

${ }^{18}$ J. O. Hirschfelder, C. F. Curtiss, and R. B. Bird, Molecular Theory of Gases and Liquids (Wiley, New York, 1954), Chap. 12.

${ }^{19}$ V. Czikklely, H. D. Forsterling, and H. Kuhn, Chem. Phys. Lett. 6, 207 (1970); and Ref. 18.

${ }^{20} \mathrm{C}$. L. Zinsser, Ph.D. thesis, University of Oklahoma, 1973.

${ }^{21}$ B. B. Johnson and W. T. Simpson, J. Chem. Phys. 65, 4246 (1976).

${ }^{22}$ Exponential, Lorentzian, Gaussian plus linear, and linear damping functions were explored.

${ }^{23}$ J. M. Turlet, P. Kottis, and M. R. Philpott, Adv. Chem. Phys. 54, 303 (1983). 